\title{
Using COPD multidimensional indices in routine clinical practice: DOSE meets all criteria
}

\author{
See linked article by Sundh et al. on pg 295
}

\section{*Niels H Chavannes ${ }^{a}$, Rupert CM Jones ${ }^{b}$, Dirkje S Postmac, Stephen Rennard ${ }^{d}$}

a Department of Public Health and Primary Care, Leiden University Medical Center, Leiden, The Netherlands

b Peninsula College of Medicine and Dentistry, Plymouth, UK; Armada Surgery, Plymouth, UK

Department of Pulmonary Medicine, University of Groningen, University Medical Center Groningen, The Netherlands

d Divison of Pulmonary, Critical Care, Sleep and Allergy, Nebraska Medical Center, Omaha, USA

*Correspondence: Associate Professor Niels H Chavannes, Leiden University Medical Center, Department of Public Health and Primary Care, Hippocratespad 21, Zone V0-P, PO Box 9600, 2300 RC Leiden, the Netherlands.

Tel: +31715268444 Fax: +31715268259

E-mail: n.h.chavannes@lumc.nl

How should we employ multidimensional indices of COPD? Ideally, they should serve to work towards 'optimal COPD care', based on the concept that here-and-now goals should be integrated with goals to improve long-term outcomes and reduce future risk.' However, this integration has (until recently) been overlooked, largely because multidimensional indices have usually been developed in selected patient groups and thus lack external validity in the community.

In this issue of the PCRJ, Josefin Sundh and colleagues present a large study assessing the merits of the DOSE (dyspnea, obstruction, smoking, exacerbations) index in predicting mortality in both primary and secondary care patients. ${ }^{2}$ This is an important paper, since it adds knowledge to the existing applicability of DOSE which previously has been shown to predict hospitalisation, respiratory failure and exacerbation risk. ${ }^{3}$ The authors investigated 1,111 COPD patients aged 34-75 years, randomly selected from 70 Swedish primary and secondary care centres. 562 patients had complete data on all DOSE index components, i.e. MRC dyspnoea scale, forced expiratory volume in 1 second $\left(F E V_{1}\right)$ expressed as percent predicted ( $F E V_{1} \%$ pred), smoking status, and exacerbation rate. Over the course of five years, 116 patients (20.6\%) died. Mortality was higher in patients with a DOSE index score $\geq 4(42.4 \%)$ than for lower scores $(11.0 \%)$ $(p<0.0001)$. Compared with a DOSE index score of $0-3$, the hazard ratio for mortality was $3.48(95 \% \mathrm{Cl} 2.32$ to 5.22 ) for a score of 4-5, and $8.00(95 \% \mathrm{Cl} 4.67$ to 13.7$)$ for a score of $6-8$. Thus, the DOSE index has now been shown to be associated with mortality in COPD patients in both primary and secondary care, and can be used to assess prognosis in addition to other clinically relevant issues.

Originally, DOSE was designed as a predictor of health status, whereas both BODE (BMI, Obstruction, Dyspnoea, Exercise) and ADO (Age, Dyspnoea, Obstruction) were derived as predictors of mortality. ADO has the disadvantage that it is affected by age; younger people with worse COPD based on MRC or FEV 1 score better than their older counterparts. This is counterintuitive, since one would expect younger people with advanced COPD to have more serious disease than older people. ${ }^{4}$ BODE has been studied extensively, yet has the major practical drawback that it requires a 6 -minute walking test. This stems from its original development in a rehabilitation setting. However, the majority of COPD patients are managed in primary care where this test is not usually performed - although it could be, provided trained personnel and a $30 \mathrm{~m}$ hallway were available.

To use any given index in primary care it should be able to identify individuals with the highest current burden of disease who are at risk of future morbidity and mortality. General criteria for the use of an index in routine clinical practice are that it should be:

(i) simple to record and calculate,

(ii) the component items should be easy to assess and record,

(iii) the component items should be clinically important in their own right,

(iv) it should be a predictor of a major measure of disease severity e.g. health status or mortality, and

(v) the index should be predictive of future exacerbations and health care consumption.

What is new in this current paper by Sundh et al.? Oga et al. previously reported on 150 patients from secondary care in Japan, and compared the DOSE, BODE and ADO indices in relation to mortality. ${ }^{5}$ They found that all three indices were significantly predictive of 5-year mortality, but that the BODE and ADO indices performed somewhat better than the DOSE index. This is hardly surprising, since BODE and $A D O$ were originally developed as indicators of mortality, whereas the DOSE index was originally designed to predict health status. The Sundh paper $^{2}$ extends this observation in a larger cohort of over 500 patients from both primary and secondary care, providing firm evidence that the DOSE index is a very good predictor of respiratory disease-specific mortality. Furthermore, the Chi-square values in the regression model used by Sundh et al. show that the DOSE index Chi-square value was 146, followed by MRC dyspnoea scale (116), air flow obstruction (91), exacerbation frequency (75.5), and smoking (54). This order was similar to that found in the original DOSE paper. ${ }^{3}$ Thus, when considering commonly used severity markers against both health status (as in the original paper) or mortality (as in the Sundh paper), the DOSE index is a stronger predictor than any of its individual items. In addition, while smoking is much vaunted as a predictor of mortality, smoking was not statistically significantly associated with mortality in the univariate analysis. The vast majority of patients with COPD are current or former smokers. However, smoking only accounts for 40$70 \%$ of the attributable risk for COPD. ${ }^{6}$ In the USA, for example, $20 \%$ of COPD patients are non-smokers, and 20\% of COPD mortality occurs in non-smokers. 'It is therefore worth investigating whether or not DOSE would be less useful in these individuals.

There are different ways to assess the value of indices in predicting mortality. In the Sundh paper ${ }^{2}$ the DOSE index score was examined in categories, whereas in the Oga paper ${ }^{5}$ the DOSE score was used as a continuous variable and Cox's proportional hazards were used. The 
data for the Sundh paper were presented as those with a score of 0-3 compared to those with a score $\geq 4$. Using Cox regression analyses the predictive value of the DOSE index was compared to other items. The methodological processes used by both papers were sound. However, for clinical use, a measure that can be used to assess change in status over time is likely to be more helpful than a measure that assesses prognosis only at one particular moment. Stratifying the DOSE at a single cut point will limit its utility in this regard. The possibility that DOSE can be used as a continuous variable, however, is one that needs to be investigated further.

Data recently presented at the 2012 International Primary Care Respiratory Group (IPCRG) World Conference in Edinburgh showed that the DOSE index but not the ADO index was a predictor of future admissions and exacerbations. ${ }^{8}$ When taking mortality as an outcome, the DOSE index has been shown to be a useful predictor of mortality in both primary and secondary care settings, yet not as strongly as the BODE and ADO indices. On the other hand, the BODE index is currently not as useful in a primary care setting where the 6-minute walk test is not routinely available. The ADO index, while simple to perform, is strongly tied to the factor 'age'. The conclusion is that the DOSE index may be the one index to meet all the criteria required of an index for use in routine primary care clinical practice, one which would provide healthcare professionals with a measure of current status and future risk in their COPD patients.

Conflicts of interest The authors declare that they have no conflicts of interest in relation to this article. NHC is an Associate Editor of the PCRJ, but was not involved in the editorial review of, nor the decision to publish, this article.
Commissioned article; not externally peer-reviewed; accepted 16th July 2012 online 26th July 2012

(C) 2012 Primary Care Respiratory Society UK. All rights reserved http://dx.doi.org/10.4104/pcrj.2012.00066

Prim Care Respir J 2012; 21(3): 245-246

\section{References}

1. Postma D, Anzueto A, Calverley $P$, et al. A new perspective on optimal care for patients with COPD. Prim Care Respir J 2011;20(2):205-09. http://dx.doi.org/ 10.4104/pcrj.2011.00041

2. Sundh J, Janson C, Lisspers K, Ställberg B, Montgomery S. The Dyspnoea, Obstruction, Smoking, Exacerbation (DOSE) index is predictive of mortality in COPD. Prim Care Respir J 2012;21:295-301. http://dx.doi.org/10.4104/ pcrj.2012.00054

3. Jones RCM, Donaldson GC, Chavannes $\mathrm{NH}$, et al. Derivation and Validation of a Composite Index of Severity in Chronic Obstructive Pulmonary Disease - The DOSE Index. Am J Resp Crit Care Med 2009;180:1189-95. http://dx.doi.org/10.1164/rccm.200902-02710C

4. Celli BR, Marin JM, Cote CG, Aguirre A, Macario CC. Prognostic assessment of patients with COPD. Lancet 2009;374(9705):1885. http://dx.doi.org/10.1016/ S0140-6736(09)62082-1

5. Oga T, Tsukino M, Hajiro T, Ikeda A, Nishimura K. Predictive properties of different multidimensional staging systems in patients with chronic obstructive pulmonary disease. Int J Chron Obstruct Pulmon Dis 2011;6:521-6. http://dx.doi.org/ 10.2147/COPD.S24420

6. Mannino DM, Buist AS. Global burden of COPD: risk factors, prevalence, and future trends. Lancet 2007;370(9589):765-73.

http://dx.doi.org/10.1016/S0140-6736(07)61380-4

7. Mannino DM, Gagnon RC, Petty TL, Lydick E. Obstructive lung disease and low lung function in adults in the United States: data from the National Health and Nutrition Examination Survey, 1988-1994. Arch Intern Med 2000;160(11):1683-98. http://dx.doi.org/ 10.1001/archinte.160.11.1683

8. Gabe-Thomas E, Jones RCM, Chavannes NH, Lee A, Hyland M, Price D. A comparison of multi-component indices of COPD severity in primary care: an UNLOCK study from the IPCRG Prim Care Respir J 2012:21(2): A7, Abstract number 153.

\section{Pandemic influenza vaccination for healthcare workers in primary care: good progress, but higher uptake required}

See linked article by Hothersall et al. on pg 302

\section{*Colin R Simpson}

a Allergy and Respiratory Research Group, Centre for Population Health Sciences, The University of Edinburgh, Edinburgh, UK

b Health Protection Scotland, Glasgow, UK

*Correspondence: Dr Colin Simpson, Allergy \& Respiratory Research Group, Centre for Population Health Sciences, The University of Edinburgh, Doorway 3, Medical School, Teviot Place, Edinburgh, EH8 9AG, UK.

Tel: +44 (0)1316514151 Fax: +44 (0)1316509119

E-mail: c.simpson@ed.ac.uk

There were three global epidemics of influenza in the last century in 1918-19, 1957-58 and 1968-69 - which resulted in considerable morbidity and mortality; the number of deaths in these pandemics has been estimated at 20 to 40 million, 1 million and 1 million, respectively. The lack of herd immunity to the novel influenza viruses implicated (i.e. H1N1, H2N2 and H3N2) is believed to have been a key factor contributing to these very high numbers of deaths. ${ }^{1}$

The World Health Organization (WHO) subsequently declared the influenza A, subtype H1N1 virus (which emerged in Mexico in March 2009) a pandemic in June 2009.2 Production of pandemic H1N1 2009 influenza monovalent vaccines began soon after confirmation of outbreaks in Europe and the USA, and in the autumn of 2009 a worldwide vaccination programme began. The pandemic vaccine was shown to be highly effective. ${ }^{3}$ However, significant global numbers of cases occurred prior to the implementation of the pandemic vaccination programme. Whilst the illness severity was in the main mild, early estimates of mortality attributable to the first pandemic wave revealed an estimated 201,200 respiratory deaths, $80 \%$ of which occurred in people aged under 65. ${ }^{4}$ Such estimates make no allowance for the illness burden seen from the same pandemic virus as it became part of the expected seasonal challenge encountered in the 2010/11 flu season and subsequently. In the UK, the severity of impact in the 2010/11 season was arguably worse, as gauged by the increased number of intensive care unit cases and deaths attributable to the H1N1 virus. ${ }^{5}$ These findings support the argument for the offer of routine influenza vaccination to healthcare workers, largely to reduce the nosocomial transmission of influenza to colleagues and vulnerable patients.

Since 1981, recommendations that healthcare workers be immunised for influenza have been in place in the UK. However, 